\title{
Inhibition of c-Myc by let-7b mimic reverses mutidrug resistance in gastric cancer cells
}

\author{
XIAOJUN YANG ${ }^{1 *}$, HUI CAI $^{1 *}$, YUHE LIANG $^{2 *}$, LIN CHEN $^{3}$, XIANGDONG WANG $^{2}$, \\ RUOHUANG SI ${ }^{1}$, KUNPENG QU ${ }^{1}$, ZEBIN JIANG ${ }^{1}$, BINGQIANG MA ${ }^{1}$, \\ CHANGFENG MIAO ${ }^{1}$, JING LI ${ }^{1}$, BIN WANG ${ }^{1}$ and PENG GAO ${ }^{1}$ \\ ${ }^{1}$ Department of General Surgery, Gansu Provincial Hospital, Lanzhou, Gansu 730000; \\ ${ }^{2}$ Department of General Surgery, the People's Hospital of Baoji City, Baoji, Shaanxi 721000; \\ ${ }^{3}$ Department of Infectious Disease, the First Hospital of Lanzhou University, Lanzhou, Gansu 730000, P.R. China
}

Received October 2, 2014; Accepted December 23, 2014

DOI: 10.3892/or.2015.3757

\begin{abstract}
Chemotherapy is one of the few effective choices for patients with advanced or recurrent gastric cancer (GC). However, the development of mutidrug resistance (MDR) to cancer chemotherapy is a major obstacle to the effective treatment of advanced GC. Additionally, the mechanism of MDR remains to be determined. In the present study, we tested $\mathrm{IC}_{50}$ of cisplating (DDP), vincristine (VCR) and 5-fluorouracil (5-FU) in SGC7901, SGC7901/DDP and SGC7901/VCR gastric cancer cells using an MTT assay. The expression of let-7b and c-Myc in these cells was detected by qPCR and western blot analysis. The relationship between let-7b and c-Myc was explored using a luciferase reporter assay. Transfection of let-7b mimic or inhibitor was used to confirm the effect of let-7b on drug sensitivity in chemotherapy via the regulation of c-Myc expression. We found that the expression of let-7b was lower in chemotherapy-resistant SGC7901/DDP and SGC7901/VCR gastric cancer cells than that in chemotherapysensitive SGC7901 cells. By contrast, the expression of c-Myc was higher in SGC7901/DDP and SGC7901/VCR cells than that in SGC7901 cells. Furthermore, we confirmed that let-7b suppresses c-Myc gene expression at the mRNA and protein levels in a sequence-specific manner, while transfection of let-7b mimic increases drug sensitivity in chemotherapyresistant SGC7901/DDP and SGC7901/VCR cells by targeting downregulation of c-Myc. In SGC7901 drug-sensitive cells, however, the sensitivity of chemotherapy was significantly decreased following let-7b inhibitor transfection. The present study results demonstrated that let-7b increases drug sensitivity
\end{abstract}

Correspondence to: Dr Peng Gao, Department of General Surgery, Gansu Provincial Hospital, 160 Donggang West Road Lanzhou, Gansu 730000, P.R. China

E-mail: gaopenglz@aliyun.com

${ }^{*}$ Contributed equally

Key words: gastric cancer, mutidrug resistance, c-Myc, let-7b in chemotherapy-resistant SGC7901/DDP and SGC7901/VCR gastric cancer cells by targeting the downregulation of c-Myc and that, let-7b mimic reverses MDR by promoting cancer stem cell differentiation controlled by double-negative autoregulatory loops (Lin28/let-7 and Myc/let-7) and a double-positive autoregulatory loop ( $\mathrm{Lin} 28 / \mathrm{Lin} 28 \mathrm{~B} / \mathrm{Myc}$ ) existing in GC cells, which remains to be confirmed.

\section{Introduction}

Gastric cancer (GC) is a leading disease in Eastern Asia (including South Korea, Japan and China). The incidence and mortality of GC in East Asian regions rank, respectively, second and third among the most common types of cancer worldwide $(1,2)$. According to World Health Organization statistics, there were 988,000 new cases of GC worldwide and 736,000 deaths in 2008. Approximately $60 \%$ of the cases were identified in East Asia (mainly China). In China, approximately two-thirds of patients develop advanced or metastatic disease, and $>50 \%$ have recurrent disease following curative surgery (3-5). For these patients, chemotherapy remains the most effective treatment of choice. However, the development of mutidrug resistance (MDR) to cancer chemotherapy is a major obstacle to the effective treatment of advanced GC (6). Moreover, the mechanism of MDR remains obscure.

$\mathrm{c}-\mathrm{Myc}$ gene is crucial in gastric carcinogenesis. The c-Myc protein is a transcription factor that regulates a large series of downstream genes. An association between c-Myc deregulation and GC has been previously demonstrated (7). c-Myc overexpression has been described in $>40 \%$ of GC (7). It encodes a helix-loop-helix leucine zipper transcription factor that dimerizes with its partner protein, Max, to transactivate gene expression $(8,9)$. c-Myc regulates several large gene families resulting in coordinated changes in cell proliferation and metabolism. c-Myc stimulates genes involved in protein biosynthesis, cancer metabolism, transcription factors, cell cycle and some microRNAs, while inhibiting the expression of other microRNAs and some tumor suppressor genes (8). The pleiotropic effects of c-Myc expression occur at the molecular and cellular level and affect almost every activity of cell life (10). 
In vivo studies have demonstrated that a single low-dose cisplatin treatment results in tumour growth retardation and a 2-fold elevation in the level of c-Myc expression $(11,12)$. This reproducible elevation in the expression of c-Myc is mirrored by reports of analysis conducted on freshly isolated colon carcinoma tissues from patients with failed cisplatin therapy (13). Although these data demonstrate that relatively low doses of cisplatin can evoke a significant increase in c-Myc expression, it is premature to suggest there is a direct link between use of cisplatin and c-Myc-modulated chemoresistance. Clearly, the mechanism for chemoresistance remains to be investigated.

MicroRNAs (miRNAs) are a group of small RNAs, which are single-stranded and consist of 19-25 nucleotides. They do not code for any protein or peptide; however, they regulate gene expression by various mechanisms. The aberrant miRNA expression and its correlation with the development and progression of cancers is an emerging field $(14,15)$. Some miRNAs regulate the formation of cancer stem cells and the acquisition of epithelial-mesenchymal transition, which are critically associated with drug resistance $(16,17)$. Moreover, some miRNAs target genes associated with drug sensitivity, resulting in the altered sensitivity of cancer cells to anticancer drugs. Findings of previous studies have also shown that the knockdown or re-expression of specific miRNAs by synthetic antisense oligonucleotides or pre-miRNAs induced drug sensitivity, leading to increased inhibition of cancer cell growth, invasion, and metastasis $(18,19)$. Those results suggested that specific targeting of miRNAs by different approaches potentially open new avenues for cancer treatment by overcoming drug resistance, thereby improving the outcome of cancer therapy.

The let-7 miRNAs are a family of 12 sequence-associated miRNAs that are distributed over eight genomic clusters and are often downregulated in cancers (20). The let-7 miRNAs function as tumor suppressors through the silencing of key oncogenes, such as RAS and MYC $(21,22)$. Findings on ovarian cancer showed that let-7i expression was significantly reduced in chemotherapy-resistant patients and in vitro reduction of let-7i expression was associated with the resistance of ovarian and breast cancer cells to cisplatin (23). By investigating drug resistance to cisplatin and 5-fluorouracil in 90 patients with GC and comparing miRNA expression of patients before and after chemotherapy, Kim et al (24) found that a high expression of let-7g indicated sensitivity to chemotherapy.

We found that c-Myc may serve as a target gene of let-7b through Targetscan and Pictar bioinformatics software. In this study, we detected the expression of let-7b and c-Myc in SGC7901 and drug-resistant SGC7901/VCR and SGC7901/ DDP GC cell lines. The aim was to determine whether let-7b regulates the sensitivity of chemotherapy to mutidrugs in GC by possibly targeting c-Myc, and to confirm our hypothesis that inhibition of c-Myc by let-7b reverses MDR in GC cells.

\section{Materials and methods}

Cell culture. The human SGC7901 gastric cancer cells were obtained from the Cell Bank of the Chinese Academy of Sciences (Shanghai, China). Its drug-resistant SGC7901/DDP and SGC7901/VCR cells were purchased from the Keygen Biotech Development Co., Ltd. (Nanjing, China). The cell lines
Table I. Primer sequences for real-time PCR.

\begin{tabular}{ll}
\hline Gene & \multicolumn{1}{c}{ Primer sequence } \\
\hline Pre-let-7 & F: TGA GGT AGT AGG TTG TGT GGT \\
& R: GGA AGG CAG TAG GTT GTA TAG \\
U6 & F: CTC GCT TCG GCA GCA CA \\
& R: AAC GCT TCA CGA ATT TGC GT \\
$c-M y c$ & F: CCC AGC GAG ACA TCT GGA AGA A \\
& R: GAG AAG CCG CTC CAC ATG CAG TC \\
Actin & F: AAG GTG AAG GTC GGA GTC AAC \\
& R: GGG GTC ATT GAT GGC AAC AATA
\end{tabular}

F, forward; R, reverse.

were cultured in RPMI-1640 (Gibco, Grand Island, NY, USA) medium with $10 \%$ fetal bovine serum (Sijiqing, Hangzhou, China), and maintained in a humidified incubator at $37^{\circ} \mathrm{C}$ with an atmosphere of $5 \% \mathrm{CO}_{2}$.

Cell viability and drug sensitivity assay. The SGC7901, SGC7901/VCR, SGC7901/DDP cells were transfected with let-7b mimic or inhibitor and control mimic. The transfected cells were seeded in 96-well plates for $24 \mathrm{~h}$ and then treated with different concentrations of cisplatin (DDP) (Qilu Pharmaceutical Co., Ltd., Shandong, China), 5-fluorouracil (5-FU) (KingYork Group Co., Ltd., Tianjin, China) and vincristine (VCR) (HuaLian Pharmaceutical Co., Ltd., Shanghai, China). After $48 \mathrm{~h}$, the cell viability was evaluated by the MTT assay according to the manual. Absorbance at $490 \mathrm{~nm}$ was measured on an ELISA reader. Dose-effect curves of anticancer drugs were drawn on semi-logarithm coordinate paper and $\mathrm{IC}_{50}$ values were determined. Each experiment was conducted in triplicate and repeated three times.

Quantitative PCR. Total RNA was extracted using TRIzol reagent (Invitrogen Inc., Carlsbad, CA, USA) and reversetranscribed using a high capacity RNA-cDNA kit (Applied Biosystems Inc., Foster City, CA, USA). cDNA was quantified on an ABI Prism 7900 sequence detection system (Applied Biosystems Inc.). PCR was performed using Power SYBR-Green PCR master mix (Applied Biosystems Inc.). The U6 small nuclear RNA was used as an internal control for let-7b. The GAPDH was used as an internal control for c-Myc. Primer sequences used are listed in Table I.

Western blot analysis. Cells were lysed in mammalian protein extraction reagent (Pierce Inc., Rockford, IL, USA) with protease inhibitor cocktail (Sigma, St. Louis, MO, USA). Following centrifugation at $5,000 \times \mathrm{g}$ for $15 \mathrm{~min}$ at $4^{\circ} \mathrm{C}$, the protein concentration was measured with a BCA protein assay kit (Pierce Inc., 23227). Total protein (15 $\mu \mathrm{g})$ was separated by $10 \%$ SDS-PAGE under denaturing conditions and transferred to PVDF membranes (Millipore Inc., Billerica, MA, USA). Membranes were blocked in 5\% non-fat milk (Bio-Rad, Hercules, CA, USA) and then incubated with the c-Myc antibody (Santa Cruz Biotechnology, Inc., Santa Cruz, CA, USA). 
A

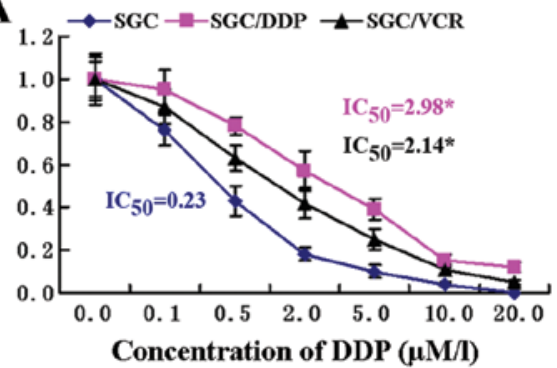

B

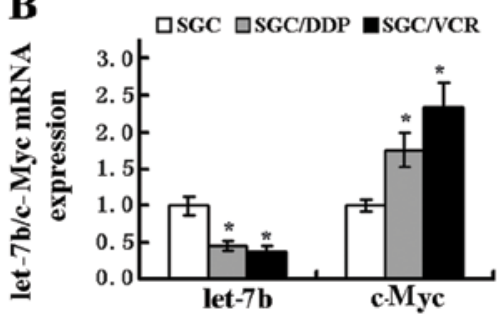

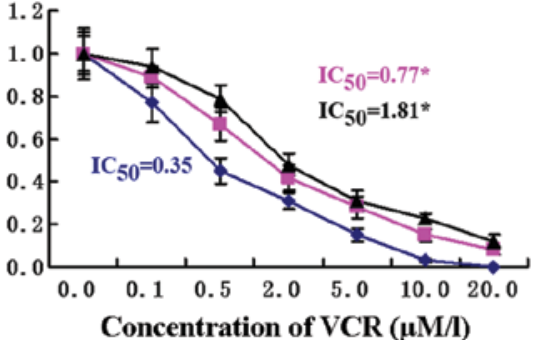

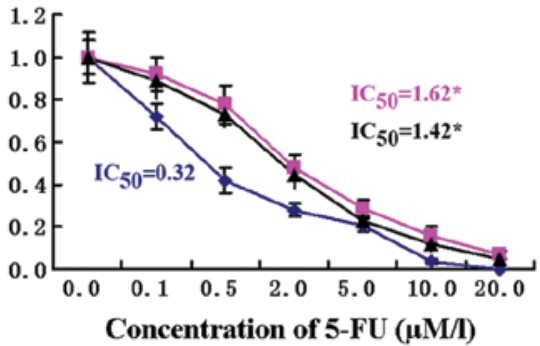

C

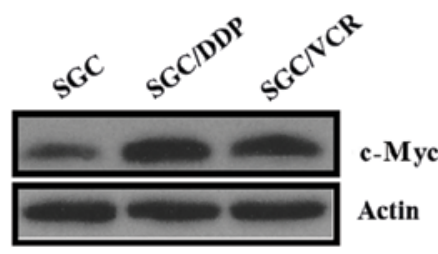

D

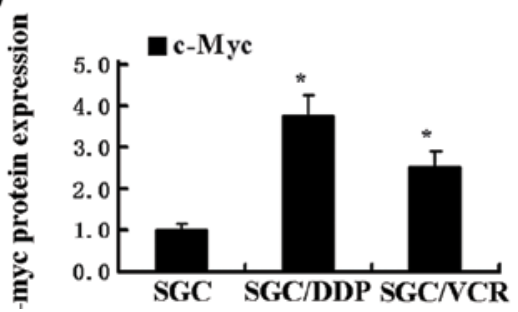

Figure 1. IC $_{50}$ of DDP, VCR, 5-FU and expression of let-7b and c-Myc in gastric cancer cells. (A) SGC7901, SGC7901/VCR, SGC7901/DDP cells were seeded in 96-well plates for $24 \mathrm{~h}$ and then treated with different concentrations of DDP, VCR and 5-FU. After $48 \mathrm{~h}$, the absorbance at $490 \mathrm{~nm}$ was measured on an ELISA reader. Dose-effect curves of anticancer drugs were drawn and $\mathrm{IC}_{50}$ values were determined; (B) Expresion of let-7b and c-Myc mRNA in SGC7901, SGC7901/VCR, SGC7901/DDP cells were analyzed by quantitive PCR ("P<0.01, vs. SGC7901 cells); (C) Expresion of c-Myc protein was evaluated by western blotting; (D) The relative expression of c-Myc protein was higher in SGC7901/DDP and SGC7901/VCR cells ("P<0.01, vs. SGC7901 cells).

After incubation with a secondary antibody conjugated with HRP (Amersham Biosciences Inc., Uppsala, Sweden) together with an HRP-conjugated primary antibody for $\beta$-actin (Sigma), immunoreactive proteins were visualized using the LumiGLO chemiluminescent substrate (Cell Signaling Technology Inc., Danvers, MA, USA). Densitometric analyses were performed using Scion Image software.

Luciferase reporter assay. The DNA encoding 3'UTR of c-Myc was PCR-amplified from human genomic DNA and cloned downstream of firefly luciferase reporter gene in pGL3-control plasmid (Promega Corp., Madison, WI, USA). SGC7901 gastric cancer cells were plated in a 24-well plate $24 \mathrm{~h}$ prior to transfection at $50 \%$ confluence. Let-7b mimic (30 $\mathrm{nM}$ ) or control mimic (Ambion Inc., Austin, TX, USA) were transfected using Lipofectamine RNAiMAX (Invitrogen Inc.). After $24 \mathrm{~h}$ post-transfection, $0.125 \mu \mathrm{g}$ of reporter vector or empty vector were transfected using FuGENE6 transfection reagent (Roche Inc., Basel, Switzerland). After 48-h reporter vector transfection, the cells were collected, and reporter assays were performed using a dual luciferase reporter assay system (Promega Corp.).

Transfection of let-7b mimic and inhibitor oligonucleotides. Pre-miR miRNA precursor and control oligos were purchased from Ambion Inc. miRCURY LNA miRNA inhibitors and control oligos were purchased from Exiqon (Vedbaek, Denmark). Transfections were performed using the Lipofectamine RNAiMAX transfection reagent (Invitrogen Inc.), and then cells were incubated in the medium containing the transfection mixture for 24-48 $\mathrm{h}$.

Statistical analysis. Data were expressed as mean $\pm \mathrm{SD}$. One-way ANOVA followed by Bonferroni correction was used to compare the data among three or more groups, followed by the Student's t-test. Statistical analyses were performed using the SPSS 15.0 software package for Windows (SPSS Inc.., Chicago, IL, USA). $\mathrm{P}<0.05$ was considered significant.

\section{Results}

$I C_{50}$ of DDP, VCR and 5-FU in SGC7901, SGC7901/DDP and SGC7901/VCR gastric cancer cells. SGC7901,SGC7901/VCR and SGC7901/DDP cells were seeded in 96-well plates for $24 \mathrm{~h}$ and then treated with different concentrations of DDP, VCR and 5-FU. After $48 \mathrm{~h}$, the cell viability was evaluated by the MTT assay according to the manual. Absorbance at $490 \mathrm{~nm}$ was measured on an ELISA reader. Dose-effect curves of anticancer drugs were drawn on semi-logarithm coordinate paper and $\mathrm{IC}_{50}$ values were determined (Fig. 1A). $\mathrm{IC}_{50}$ of DDP in SGC7901, SGC7901/DDP and SGC7901/VCR cells were $0.23,2.98$ and $2.14 \mu \mathrm{M} / 1$, respectively. $\mathrm{IC}_{50}$ of VCR in SGC7901, SGC7901/DDP and SGC7901/VCR cells were $0.35,0.77$ and $1.18 \mu \mathrm{M} / 1$, respectively. $\mathrm{IC}_{50}$ of $5-\mathrm{FU}$ in SGC7901, SGC7901/DDP and SGC7901/VCR cells were $0.32,1.62$ and $1.42 \mu \mathrm{M} / 1$, respectively.

Expression of let-7b and c-Myc in SGC7901, SGC7901/ DDP and SGC7901/VCR gastric cancer cells. To investigate the potential role of let-7b on MDR in GC, the expression of let-7b in SGC7901, SGC7901/DDP and SGC7901/VCR cells was evaluated by qPCR. A significant difference was observed between SGC7901 and the drug-resistant SGC7901/DDP and SGC7901/VCR cells (Fig. 1B). The expression of c-Myc mRNA was increased in SGC7901/DDP and SGC7901/VCR cells compared with that of SGC7901 cells (Fig. 1B). To confirm this phenomenon, we also performed western blot analysis for $\mathrm{c}-\mathrm{Myc}$ protein. The protein levels of c-Myc were higher in 
A

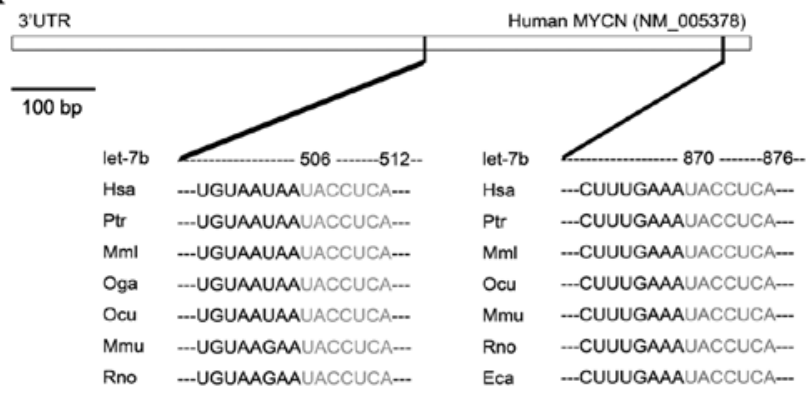

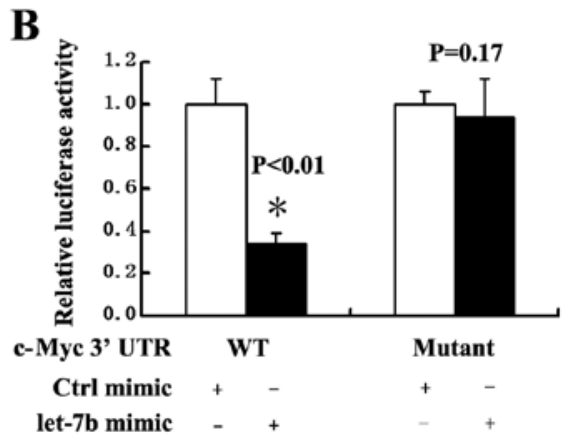

Figure 2. Let-7b regulates the expression of c-Myc by directly targeting 3'UTR. (A) A conserved let-7 binding site in the c-Myc 3'UTR was detected by TargetScan. (B) The c-Myc 3'UTR reporter assay showed that transfection of a let-7b mimic significantly reduced the luciferase activity in the wild-type but not the let-7 binding site mutant c-Myc 3'UTR reporters $\left({ }^{*} \mathrm{P}<0.01\right)$.

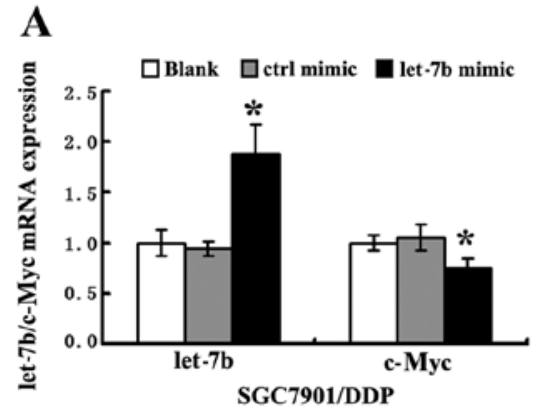

C

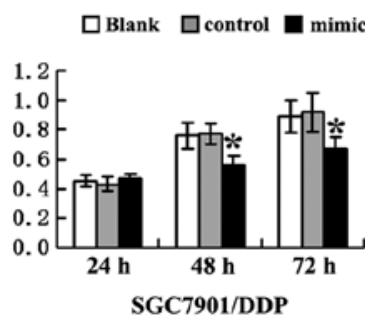

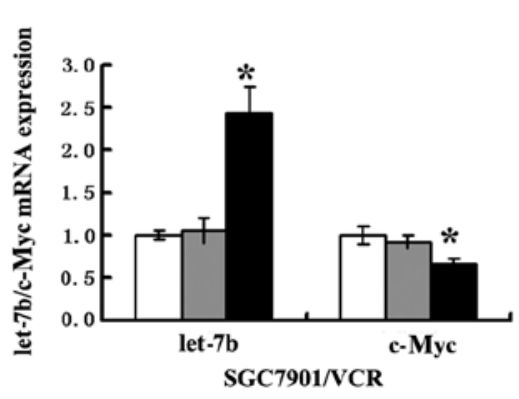

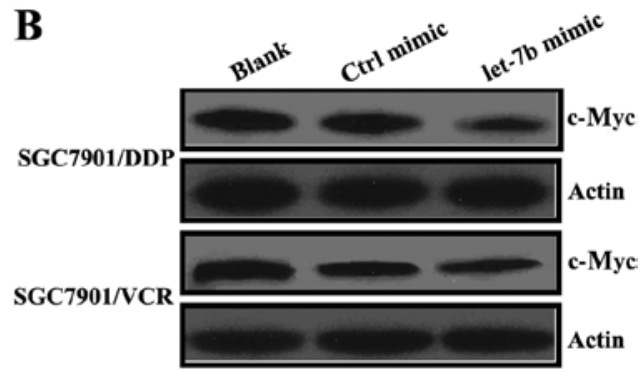

Figure 3. Transfection of let-7b mimic downregulates the expression of c-Myc both at mRNA and protein levels in SGC7901/DDP and SGC7901/VCR cells, increasing the sensitivity of chemotherapy. (A) Expression of let-7b was significantly higher in the two cells after let-7b mimic transfection. By contrast, the expression of c-Myc was significantly lower in the two cells after let-7b mimic transfection ( $\left.{ }^{*}<0.05\right)$. (B) The results of western blotting show that transfection of let-7b mimic significantly downregulates the expression of c-Myc protein in the two cells. (C) MTT assay show that the cell viability was reduced significantly in the two cells after transfection with the let-7b mimic at 48 and $72 \mathrm{~h}$ as compared with the control mimic ("P<0.05). (D) IC ${ }_{50}$ values of $\mathrm{DDP}, \mathrm{VCR}$ and 5 -FU were determined in the two cells by MTT assay and were downregulated by let-7b mimic transfection $\left({ }^{*} \mathrm{P}<0.05\right)$.

SGC7901/DDP and SGC7901/VCR cells than that in SGC7901 cells (Fig. 1C and D). Taken together, we demonstrated that there is a potential correlation between let-7b and c-Myc.

Let-7b directly targets the 3'UTR of $c-M y c$. We predicted that there was a conserved let-7 binding site in the c-Myc 3'UTR by TargetScan (Fig. 2A). This hypothesis was confirmed experimentally. To determine whether let-7b regulation to $c-M y c$ gene depends on its binding sites of 3'UTR sequences on the target genes, we constructed reporter plasmids with wild-type or mutant let-7b binding sites from 3'UTR of c-Myc gene inserted in the downstream sequence of the luciferase gene. A reporter assay was performed by co-transfecting SGC7901/DDP cells with wild-type or mutant reporter plasmids and let-7b mimic or control oligos. Let-7b potently decreased the luciferase activity of wild-type reporter plasmid examined in this study, whereas it
D
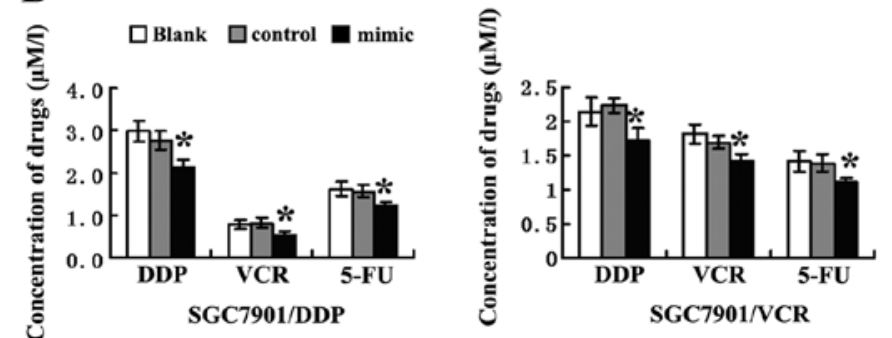

had no effect on the mutant forms (Fig. 2B). We concluded that let-7b suppressed $c-M y c$ gene expression in a sequence-specific manner and the suppression depends on let-7b binding sites within 3'UTR sequences of the $c-M y c$ genes.

Transfection of let-7b mimic downregulates the expression of $c-M y c$ and increases the sensitivity of chemotherapy in SGC7901/DDP and SGC7901/VCR cells. To investigate whether let-7b could regulate c-Myc expression, let-7b mimic and control mimic, respectively, were transfected in SGC7901/DDP and SGC7901/VCR cells. The expression of let-7b and c-Myc in the two cell lines was detected by qPCR. As shown in Fig. 3A, the expression of let-7b was significantly higher in let-7b mimic-transfected cells than that in control mimic-transfected cells. However, the expression of c-Myc was significantly lower in let-7b mimic-transfected cells than that in the control 
A

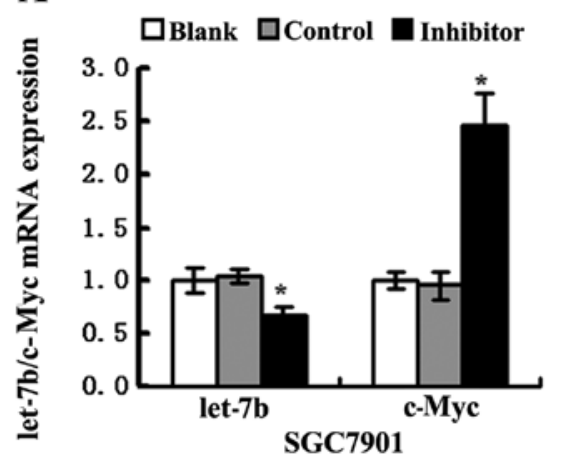

C

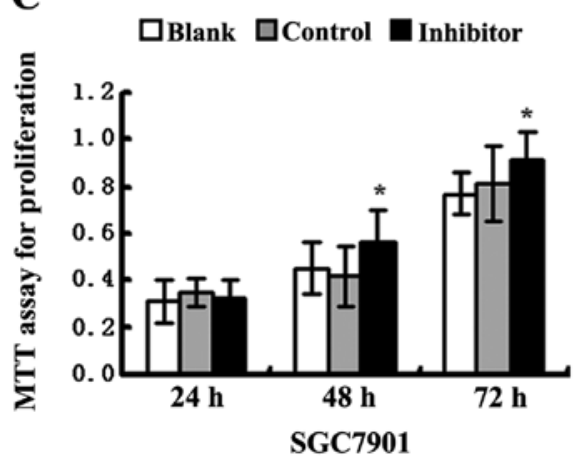

B

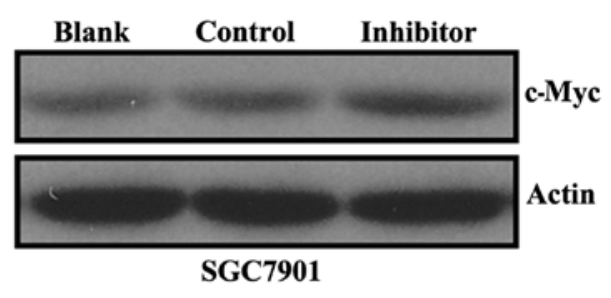

D

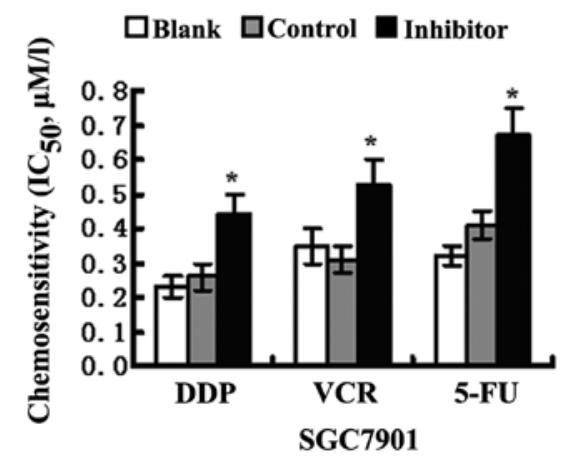

Figure 4. Transfection of let-7b inhibitor upregulates the expression of c-Myc at the mRNA and protein levels in SGC7901 cells and decreases the sensitivity of chemotherapy. (A) Expression of let-7b was significantly decreased in SGC7901 cells after let-7b inhibitor transfection. By contrast, the expression of c-Myc was significantly higher after let-7b mimic transfection. ( $\mathrm{P}<0.05)$. (B) The results of western blotting show that transfection of let-7b inhibitor significantly upregulates the expression of c-Myc protein. (C) MTT assay show the cell viability was increased significantly in SGC7901 cells after transfection with the let-7b mimic at 48 and $72 \mathrm{~h}$ as compared with the control mimic ( $\mathrm{P}<0.05$ ). (D) $\mathrm{IC}_{50}$ values of DDP, VCR and 5-FU were determined by MTT assay and were upregulated by let-7b inhibitor transfection (" $\mathrm{P}<0.05)$.

mimic-transfected cells. From the results of western blotting, we found that transfection of let-7b mimic significantly downregulated the expression of c-Myc protein in the two cells (Fig. 3B). Therefore, we concluded that let-7b suppresses $c-M y c$ gene expression at the mRNA and protein levels.

To determine whether transfection of let-7b mimic affects cell proliferation in SGC7901/DDP and SGC7901/VCR cells, metabolic activity at 24,48 and $72 \mathrm{~h}$ after transfection was determined by MTT assay. The cell viability was reduced significantly in the two cells after transfection with the let-7b mimic at 48 and $72 \mathrm{~h}$ as compared with the control mimic (Fig. 3C, $\mathrm{P}<0.05$ ).

To explore the role of c-Myc on the sensitivity of chemotherapy in GC cells, SGC7901/VCR and SGC7901/DDP cells were transfected with let-7b or control mimic. The transfected cells were seeded in 96-well plates for $24 \mathrm{~h}$ and then treated with different concentrations of DDP, VCR and 5-FU. After $48 \mathrm{~h}$, the cell viability was evaluated by the MTT assay according to the manual, dose-effect curves of anticancer drugs were drawn on semi-logarithm coordinate paper and $\mathrm{IC}_{50}$ values were determined. As shown in Fig. 3D, the $\mathrm{IC}_{50}$ valus of DDP, VCR and 5-FU were $2.13 \pm 0.18,0.54 \pm 0.08$ and $1.23 \pm 0.09 \mu \mathrm{M} / 1$ in let-7b mimic-transfected SGC7901/DDP cells, and were $2.76 \pm 0.22,0.81 \pm 0.12$ and $1.57 \pm 0.16 \mu \mathrm{M} / 1$ in control mimic-transfected SGC7901/DDP cells. For SGC7901/ VCR cells, the $\mathrm{IC}_{50}$ of DDP, VCR and 5-FU were 1.71 \pm 0.19 , $1.41 \pm 0.11$ and $1.11 \pm 0.05 \mu \mathrm{M} / 1$ in let-7b mimic-transfected cells, and were $2.23 \pm 0.11,1.69 \pm 0.09$ and $1.39 \pm 0.12 \mu \mathrm{M} / 1$ in control mimic transfected cells, respectively. Thus, the two drug-resistant GC cells showed the sensitivity of chemotherapy to be significantly increased in let-7b mimic-transfected cells at the 3rd day after transfection.

Transfection of let-7b inhibitor upregulates the expression of $c-M y c$ and decreases the sensitivity of chemotherapy in SGC7901 cells. For loss-of-function experiments, let-7b inhibitor was used to block endogenous let-7b expression in SGC7901 cell lines. The expression of let-7b and c-Myc was detected by qPCR. As shown in Fig. 4A, the expression of let-7b was significantly lower in let-7b inhibitor-transfected cells than that in control mimic-transfected cells. However, the expression of c-Myc was significantly higher in let-7b inhibitor-transfected cells than that in control mimic-transfected cells. From the results of western blotting, we found that the transfection of let-7b inhibitor significantly increased the expression of c-Myc protein in SGC7901 cells (Fig. 4B). We also confirmed that transfection of let-7b inhibitor enhances SGC7901 cell proliferation at 48 and $72 \mathrm{~h}$ after transfection by MTT assay (Fig. 4C, $\mathrm{P}<0.05$ ).

The sensitivity of chemotherapy in SGC7901 cells was also detected by MTT assay following transfection with let-7b inhibitor or control mimic. As shown in Fig. 4D, the $\mathrm{IC}_{50}$ values of DDP, VCR and 5-FU were $0.44 \pm 0.06,0.53 \pm 0.07$ and $0.67 \pm 0.08 \mu \mathrm{M} / 1$ in let-7b inhibitor-transfected SGC7901 cells, 


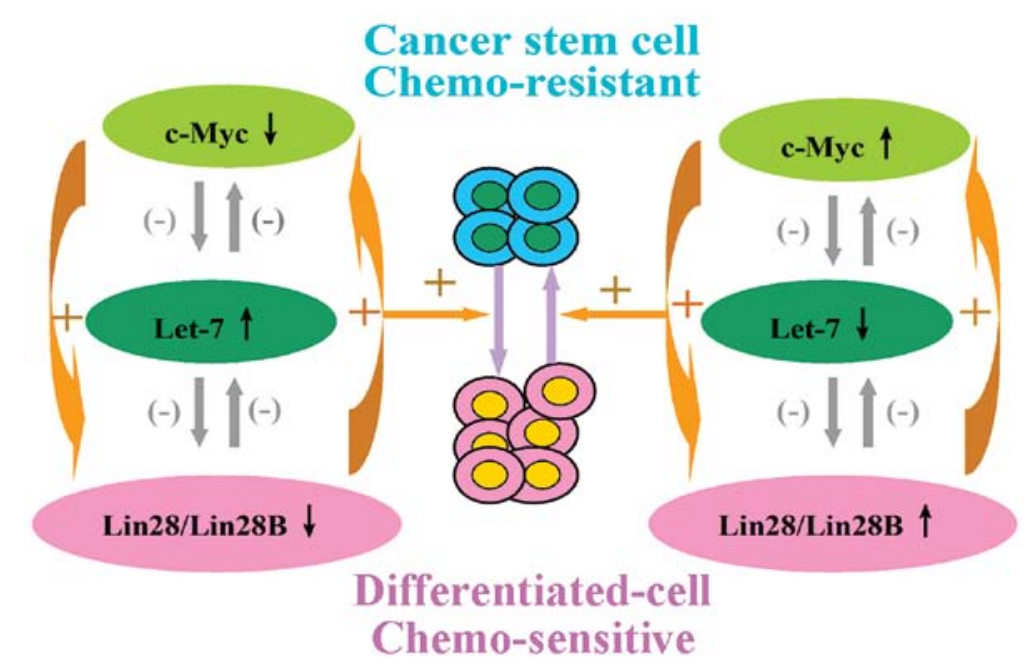

Figure 5. A possible model. Our present findings together with previous studies support a model in which double-negative autoregulatory loops (Lin28/let-7 and Myc/let-7) and a double-positive autoregulatory loop (Lin28/Lin28B/Myc) existing in gastric cancer cells may control cancer stem cell differentiation and regulate MDR.

and were $0.26 \pm 0.04,0.31 \pm 0.04$ and $0.41 \pm 0.04 \mu \mathrm{M} /$ in control mimic-transfected cells, respectively. We demonstrated that in drug-sensitive GC cells, the sensitivity of chemotherapy was significantly decreased following let-7b inhibitor transfection.

\section{Discussion}

Chemotherapy is an important therapeutic strategy for advanced or recurrent gastric cancer (GC) treatment. However, chemotherapy fails to eliminate all tumor cells because of intrinsic or acquired MDR, which is the most common cause of tumor recurrence $(25,26)$, and the greatest obstacle for the effective treatment of GC (6). The underlying mechanisms of cellular resistance in cancer cells to these DNA-damaging anticancer drugs have been broadly explored, but have not been fully characterized $(27,28)$. P-gp was the first molecule identified as a modulator of MDR. Subsequently, various other molecules were shown to be involved, including transporters that eject anticancer drugs from cells, such as MDR-associated protein (MRP) (29), genes regulating apoptosis, such as p53 (30), telomere-binding protein, such as TRF2(31), RhoE GTPase (32) and transcription factors, such as CDX2 (33) and c-Myc (13).

miRNAs have also beeen considered to be involved in the field of cancer MDR investigations. miRNAs are a class of small, non-coding RNA molecules that repress protein expression through imperfect binding to sequences in the 3'UTR of target mRNAs, Seven miRNAs were found to be significantly and differentially expressed in tumors from platinum-sensitive vs. platinum-resistant patients. These seven miRNAs included miR-27a, miR-23a, miR-30c, let-7g, miR-199a-3p, miR-378 and miR-625, which were overexpressed in platinum-resistant patients (34). Results of a recent study suggested that miRNA-200c regulates the sensitivity of chemotherapy to cisplatin (DDP) in SGC7901/DDP gastric cancer cells by directly targeting $\mathrm{RhoE}$ (32). Chen et al (35) transfected miRNA-200c into SGC7901/DDP gastric cancer cells, which increased sensitivity to DDP, 5-fluorouracil, paclitaxel and doxorubicin.
In the present study, we found that the expression of let-7b was lower in chemotherapy-resistant SGC7901/DDP and SGC7901/ VCR gastric cancer cells than that in chemotherapy-sensitive SGC7901 cells. By contrast, the expression of c-Myc was higher in SGC7901/DDP and SGC7901/VCR cells than that in SGC7901 cells. Using TargetScan and luciferase reporter assay, we confirmed that let-7b suppresses $c-M y c$ gene expression in a sequence-specific manner and this suppression depends on let-7b binding sites within 3'UTR sequences of the c-Myc genes. Furthermore, we have demonstrated that transfection of let-7b mimic increases drug sensitivity in chemotherapyresistant SGC7901/DDP and SGC7901/VCR cells by targeting the downregulation of c-Myc. For loss-of-function experiments, the results suggest that in drug-sensitive SGC7901 cells, the sensitivity of chemotherapy was significantly decreased after let-7b inhibitor transfection.

An increasing number of reports have suggested that let-7 is poorly expressed in a variety of human tumors and a reduced let-7 level results in the overexpression of let-7-responsive genes in tumors, including CyclinD, RAS, Myc and Lin28 / Lin28B (36-39). According to our previous report, a doublenegative feedback regulating loop of Lin28 and let-7 exists in tumor cells and controls ALDH1+ cancer stem cells (40). let-7 interacts with two iPS genes, Myc and Lin28, and these autoregulatory loops of let-7/Myc (41) and let-7/Lin28 (40) may control stem cell self-renewal and differentiation. High levels of Lin28 and Myc or a low level of let-7 may promote the conversion of epithelial cells to a more undifferentiated stage and maintain tumor cells in this stem-like stage $(40,42)$. A recent study found that Lin28B enhances n-Myc levels and induces neuroblastoma, suggesting that $\mathrm{n}-\mathrm{Myc}$ is a key target of Lin28B (43). Moreover, the $c-M y c$ and $n-M y c$ oncogenes are positive regulators of Lin 28 and Lin28B, respectively $(44,45)$.

Emerging evidence suggests that cancer stem cells (CSCs) may play critical roles in drug resistance and ultimately recurrence (46-50). The present findings together with those of previous reports $(40,41,43-45)$ support a model in which double-negative autoregulatory loops (Lin28/let-7 and Myc/let-7) and a double-positive autoregulatory loop 
(Lin28/Lin28B/Myc) existing in GC cells may control cancer stem cell differentiation and regulate MDR (Fig. 5).

In conclusion, the present study results have demonstrated that, let-7b increases drug sensitivity in chemotherapy-resistant SGC7901/DDP and SGC7901/VCR gastric cancer cells by targeting downregulation of c-Myc and that, let-7b mimic reverses MDR by promoting cancer stem cell differentiation controlled by double-negative autoregulatory loops (Lin28/ let-7 and Myc/let-7) and a double-positive autoregulatory loop (Lin28/ Lin28B/Myc) existing in GC cells, which remains to be confirmed. These results suggest that let-7b plays key roles in MDR of GC. By downregulating Lin28 and c-Myc and promoting cancer stem cell differentiation, let-7b may function as a chemotherapy enhancer in GC. This role in regulating GC cell MDR signifies an opportunity to develop novel cancer therapies. Therefore, it is presumed that enforced expression of let-7b may improve the chemotherapy efficiency of patients with GC with a low let-7b expression.

\section{Acknowledgements}

This study was supported, in whole or in part, by GWGL201347(HC), and Natural Science Foundation of Gansu Province (145RJZA117, HC). We are greatly indebted to Dr Youcheng Zhang (Lanzhou University Second Hospital) and Lin Zhang (University of Pennsylvania School of Medicine) for the technical assistance.

\section{References}

1. Lee JH, Kim KM, Cheong JH and Noh SH: Current management and future strategies of gastric cancer. Yonsei Med J 53: 248-257, 2012.

2. Hudler P: Genetic aspects of gastric cancer instability. Sci World J: 2012: 761909, 2012.

3. Verdecchia A, Corazziari I, Gatta G, Lisi D, Faivre J and Forman D (EUROCARE Working Group): Explaining gastric cancer survival differences among European countries. Int $\mathrm{J}$ Cancer 109: 737-741, 2004.

4. Bonenkamp JJ, Hermans J, Sasako M, et al: Extended lymphnode dissection for gastric cancer. N Engl J Med 340: 908-914, 1999.

5. Kaneko S and Yoshimura T: Time trend analysis of gastric cancer incidence in Japan by histological types, 1975-1989. Br J Cancer 84: 400-405, 2001.

6. Fan D and Liu X: New progresses in researches on multidrug resistance in gastric cancer. Chin J Digest 20: 77-78, 2000.

7. Milne AN, Sitarz R, Carvalho R, Carneiro F and Offerhaus GJ: Early onset gastric cancer: on the road to unraveling gastric carcinogenesis. Curr Mol Med 7: 15-28, 2007.

8. Eilers M and Eisenman RN: Myc's broad reach. Genes Dev 22: 2755-2766, 2008.

9. Herkert B and Eilers M: Transcriptional repression: the dark side of myc. Genes Cancer 1: 580-586, 2010.

10. Miller MD, Thomas SD, Islam A, Muench D and Sedoris K: C-Myc and cancer metabolism. Clin Cancer Res 18: 5546-5553, 2012 .

11. Walker TL, White JD, Esdale WJ, Burton MA and DeCruz EE Tumour cells surviving in vivo cisplatin chemotherapy display elevated cmyc expression. Br J Cancer 73: 610-614, 1996.

12. Marazzi L, Parodi MT, Martino DD, Ferrari S and Tonini GP: Coordinate change of c-myc, transferrin receptor and $\mathrm{H} 3$ gene expression precedes induction of haemoglobin-producing cells of the leukaemia K562 cell line treated with cisdiamminedichloroplatinum II. Anticancer Res 11: 947-952, 1991.

13. Kashani-Sabet M, Lu Y, Leong L, Haedicke K and Scanlon KJ: Differential oncogene amplification in tumour cells from a patient treated with cisplatin and 5-FU. Eur J Cancer 26: 383-390, 1990

14. Vandenboom TG II, Li Y, Philip PA and Sarkar FH: MicroRNA and cancer: tiny molecules with major implications. Curr Genomics 9: 97-109, 2008.
15. Iorio MV and Croce CM: MicroRNAs in cancer: small molecules with a huge impact. J Clin Oncol 27: 5848-5856, 2009.

16. Peter ME: Regulating cancer stem cells the miR way. Cell Stem Cell 6: 4-6, 2010.

17. Adam L, Zhong M, Choi W, et al: miR-200 expression regulates epithelial-to-mesenchymal transition in bladder cancer cells and reverses resistance to epidermal growth factor receptor therapy. Clin Cancer Res 15: 5060-5072, 2009.

18. Bourguignon LY, Spevak CC, Wong G, Xia W and Gilad E: Hyaluronan-CD44 interaction with protein kinase C(epsilon) promotes oncogenic signaling by the stem cell marker Nanog and the Production of microRNA-21, leading to down-regulation of the tumor suppressor protein PDCD4, anti-apoptosis, and chemotherapy resistance in breast tumor cells. J Biol Chem 284: 26533-26546, 2009.

19. Blower PE, Chung JH, Verducci JS, et al: MicroRNAs modulate the chemosensitivity of tumor cells. Mol Cancer Ther 7: 1-9, 2008.

20. Roush S and Slack FJ: The let-7 family of microRNAs. Trends Cell Biol 18: 505-516, 2008.

21. Lee YS and Dutta A: The tumor suppressor microRNA let-7 represses the HMGA2 oncogene. Genes Dev 21: 1025-1030, 2007.

22. Kumar MS, Lu J, Mercer KL, Golub TR and Jacks T: Impaired microRNA processing enhances cellular transformation and tumorigenesis. Nat Genet 39: 673-677, 2007.

23. Yang N, Kaur S, Volinia S, et al: MicroRNA microarray identifies Let-7i as a novel biomarker and therapeutic target in human epithelial ovarian cancer. Cancer Res 68: 10307-10314, 2008.

24. Kim CH, Kim HK, Rettig RL, et al: miRNA signature associated with outcome of gastric cancer patients following chemotherapy. BMC Med Genomics 4: 79, 2001.

25. Broxterman HJ, Gotink KJ and Verheul HM: Understanding the causes of multidrug resistance in cancer: a comparison of doxorubicin and sunitinib. Drug Resist Updat 12: 114-126, 2009.

26. Fojo T: Multiple paths to a drug resistance phenotype: mutations, translocations, deletions and amplification of coding genes or promoter regions, epigenetic changes and microRNAs. Drug Resist Updat 10: 59-67, 2007.

27. Gatti L and Zunino F: Overview of tumor cell chemoresistance mechanisms. Methods Mol Med 111: 127-148, 2005.

28. Fan D, Zhang X, Chen X, et al: Bird's-eye view on gastric cancer research of the past 25 years. J Gastroenterol Hepatol 20: 360-365, 2005.

29. Chuman Y, Sumizawa T, Takebayashi Y, et al: Expression of the multidrug resistance associated protein (MRP) gene in human colorectal, gastric and non-small-cell lung carcinomas. Int J Cancer 66: 274-279, 1996.

30. Matsuhashi N, Saio M, Matsuo A, Sugiyama Y and Saji S: The evaluation of gastric cancer sensitivity to 5-FU/CDDP in terms of induction of apoptosis: Time- and p53 expression-dependency of anti-cancer drugs. Oncol Rep 14: 609-615, 2005.

31. Ning H, Li T, Zhao L, et al: TRF2 promotes multidrug resistance in gastric cancer cells. Cancer Biol Ther 5: 950-956, 2006.

32. Chang L, Guo F, Wang Y, et al: MicroRNA-200c regulates the sensitivity of chemotherapy of gastric cancer SGC7901/DDP cells by directly targeting RhoE. Pathol Oncol Res 20: 93-98, 2014

33. Yan LH, Wang XT, Yang J, et al: Reversal of multidrug resistance in gastric cancer cells by CDX2 downregulation. World $\mathrm{J}$ Gastroenterol 19: 4155-4165, 2013.

34. Eitan R, Kushnir M, Lithwick-Yanai G, et al: Tumor microRNA expression patterns associated with resistance to platinum based chemotherapy and survival in ovarian cancer patients. Gynecol Oncol 114: 253-259, 2009.

35. Chen Y, Zuo J, Liu Y, Gao H and Liu W: Inhibitory effects of miRNA-200c on chemotherapy-resistance and cell proliferation of gastric cancer SGC7901/DDP cells. Chin J Cancer 29: 1006-1011, 2010

36. Schultz J, Lorenz P, Gross G, Ibrahim S and Kunz M: MicroRNA let-7b targets important cell cycle molecules in malignant melanoma cells and interferes with anchorage-independent growth. Cell Res 18: 549-557, 2008.

37. Sampson VB, Rong NH, Han J, et al: MicroRNA let-7a downregulates MYC and reverts MYC-induced growth in Burkitt lymphoma cells. Cancer Res 67: 9762-9770, 2007.

38. Johnson SM, Grosshans H, Shingara J, et al: RAS is regulated by the let-7 microRNA family. Cell 120: 635-647, 2005.

39. RybakA, Fuchs H, Smirnova L, et al: A feedback loop comprising lin-28 and let-7 controls pre-let-7 maturation during neural stem-cell commitment. Nat Cell Biol 10: 987-993, 2008. 
40. Yang XJ, Lin XJ, Zhong XM, et al: Double negative feedback loop between reprogramming factor LIN28 and microRNA let-7 regulates aldehyde dehydrogenase 1-positive cancer stem cells. Cancer Res 70: 9463-9472, 2010.

41. Chang TC, Zeitels LR, Hwang HW, et al: Lin-28B transactivation is necessary for Myc-mediated let-7 repression and proliferation. Proc Natl Acad Sci USA 106: 3384-3389, 2009.

42. Cotterman R and Knoepfler PS: N-Myc regulates expression of pluripotency genes in neuroblastoma including lif, klf2, klf4, and lin28b. PLoS One 4: e5799, 2009.

43. Molenaar JJ, Domingo-Fernández R, Ebus ME, et al: LIN28B induces neuroblastoma and enhances MYCN levels via let-7 suppression. Nat Genet 44: 1199-1206, 2012.

44. Laurenti E, Varnum-Finney B, Wilson A, et al: Hematopoietic stem cell function and survival depend on c-Myc and N-Myc activity. Cell Stem Cell 3: 611-624, 2008.

45. Stanton BR, Perkins AS, Tessarollo L, Sassoon DA and Parada LF: Loss of Nmyc function results in embryonic lethality and failure of the epithelial component of the embryo to develop. Gene Dev 6: 2235-2247, 1992.
46. Konopleva M, Tabe Y, Zeng Z and Andreeff M: Therapeutic targeting of micro environmental interactions in leukemia: mechanisms and approaches. Drug Resist Updat 12: 103-113, 2009.

47. Voulgari A and Pintzas A: Epithelial-mesenchymal transition in cancer metastasis: mechanisms, markers and strategies to overcome drug resistance in the clinic. Biochim Biophys Acta 1796: 75-90, 2009.

48. Wang Z, Li Y, Banerjee S and Sarkar FH: Emerging role of Notch in stem cells and cancer. Cancer Lett 279: 8-12, 2009.

49. Wang ZW, Li YW, Ahmad A, et al: Targeting miRNAs involved in cancer stem cell and EMT regulation: an emerging concept in overcoming drug resistance. Drug Resist Updat 13: 109-118, 2010.

50. Ahmed N, Abubaker K, Findlay J and Quinn M: Cancerous ovarian stem cells: obscure targets for therapy but relevant to chemoresistance. J Cell Biochem 114: 21-34, 2013. 